\title{
A New Approach to Ultrasound Guided Radio-Frequency Needle Placement
}

\author{
Claudio Alcérreca ${ }^{1,2}$, Jakob Vogel ${ }^{2}$, Marco Feuerstein $^{2}$ and Nassir Navab ${ }^{2}$ \\ ${ }^{1}$ Image Analysis a. Visualization Lab, CCADET UNAM, 04510 México City, México \\ ${ }^{2}$ Chair for Computer Aided Medical Procedures (CAMP), TU München, Germany \\ Email: claudio@uxmcc2.iimas.unam.mx
}

\begin{abstract}
The transformation from an ultrasound (US) image plane to the coordinate system of a position sensor attached to the US transducer can be computed with US calibration algorithms. This knowledge can be used in many applications, including freehand 3D US and US guided surgical navigation. We present a software system assisting the surgeon to position a radio-frequency ( $\mathrm{RF}$ ) tumor ablation needle using augmented ultrasound, thus simplifying the treatment by (1) dividing it into two simple consecutive tasks, lesion finding and needle placement, and (2) relating the needle to the US plane at any time.
\end{abstract}

\section{Introduction}

Since their introduction into clinical practice, ultrasound (US) systems gained wide acceptance among physicians. With US images evolving in sharpness and overall quality, the combination of tracking with US systems allows the development of interactive navigation systems, which facilitate common minimally invasive treatments, for instance radio-frequency (RF) tumor ablation. The latter uses a high power and high frequency generator connected to electrodes in the tip of special needles. Electricity flows from these electrodes to a foil pad, usually attached to the patient's back or thighs [1], causing local necrosis in cells around the tip of the needles. This way, the tumor can be treated without the need of open surgery or other highly invasive procedures.

Currently, RF is used in every part of the body. In order to place the tip of the needle in the center of the lesion, US imaging is commonly used for guidance. In this case, the surgeon tries to visualize the tumor and the needle in the same US plane. The needle, however, is not visible in the US image before insertion, making it difficult to predict the tumor location relative to the needle tip and its orientation. Additionally, the surgeon needs to precisely handle the US transducer and the needle at the same time.

\section{Related work}

Many authors have published articles on freehand US calibration. Prager et al. suggested the single wall method [2], where a tracked probe is used to scan the 
planar bottom of a water basin. This surface is clearly visible in the US image as a single line and may be recovered automatically using image processing algorithms. This approach has two major advantages over other procedures [3]. First, the calibration phantom does not need to be carefully manufactured. Secondly, US images containing a straight line can be processed automatically with high accuracy in real time. Lang $\varnothing$ enhanced this method by adding a nylon mesh to the setup, which produces even sharper features in the images [4].

Some surgical navigation systems have been developed to support needle placement procedures. BrainLAB ${ }^{1}$ has a commercial system that can be used to guide a needle using preoperative 3D data (CT, MRI, or PET). The main problem with this approach is the need of a correct registration to match the preoperative studies with the anatomy of the patient. This is usually done by manually selecting corresponding points. In contrast to this solution, Ultraguide 1000 [5] relies on US data for guided needle placement, without the need for data registration. Using an electro-magnetic tracking system, it however requires additional cabling and is prone to distortions. Another approach proposed by Khamene et al. [6] uses a head mounted display with augmented reality capabilities. This method gives a very intuitive navigation, but requires specific and expensive hardware.

\section{Methods}

We developed a US calibration and needle placement system, which makes use of the CAMPAR framework [7]. We use an infrared based optical tracking system with four ARTtrack2 cameras, which defines the world coordinate system. For US image acquisition we use a Picker Computer Sonograph CS 9300 with a curvilinear 3.5-MHz transducer. After calibration of the US probe, needle tip, and an additional tracked camera, we are able to obtain all necessary transformations to relate every tracked object to the world coordinate system (Fig. 1, left). For testing, we first obtained a calibration transformation and applied it to our needle placement component. In the next sections, we describe our system in more detail.

\subsection{Automatic line detection}

Selecting a suitable water basin is a major factor for single wall US calibration, since many materials create reflections in the image and thus complicate the automatic detection of the basin's ground. We used a clay pot for our first experiments. Later on, as we required more space, we switched to a plastics box holding a planar nylon membrane stretched over an aluminum frame [4]. In both cases, our system is able to automatically detect the bottom plane using an algorithm proposed by Prager et al. [2]. Lines are detected in two separate steps: First, the algorithm attempts to find feature points along certain predefined vertical scan lines. These one-dimensional signals are smoothed using a Gaussian

\footnotetext{
1 http://www $\cdot$ brainlab.com
} 
filter kernel, then median-filtered, and finally differentiated. All positions above a predefined threshold are kept. In a second step, an implementation of the random sample consensus algorithm (RANSAC) attempts to match several candidate lines through these feature points [8]. The final line is chosen from these candidates taking into account the number of features supporting the respective line and the proximity to the line detected in the last frame.

\subsection{Ultrasound calibration procedure}

First, a temporal calibration can be performed. In principle, we adopted the protocol suggested by Treece et al. [9]. The full pose data of the probe is recorded and principal components analysis (PCA) is applied to find the major axis of movement independent from the orientation of the world coordinate system.

Next, we compute the spatial transformation from the US coordinate system to the probe system. The user therefor needs to perform a series of motions to cover all six degrees of freedom. At the same time, tracking data and line positions are stored. The calibration parameters are obtained using the Levenberg-Marquardt optimizer. We are using a second position matrix to relax the system. Originally, we recorded data continuously, thus working with about 2,000 to 3,000 samples. As the results were not as good as expected, we switched to the protocol proposed by Hsu et al. that requires human interaction to select suitable poses [10].

\subsection{Needle placement}

The US guided needle placement application helps the surgeon to insert the tip of an RF needle into a precise region of interest, defined by the user, in a similar way as the Ultraguide system [5], which has been proven to have good accuracy. Unlike Ultraguide, our system provides the means to automatically calibrate an RF needle with high accuracy. Additionally, various 3D rendering techniques are utilized. It has four different views (Fig. 1, right):

- Ultrasound view: The user sees the US video, with the option to freeze an image and define a spherical target. Using the US calibration results, the system maps the defined target to the world coordinate system.

- World interactive view: The surgeon can see the needle and the sphere target in a virtual environment. This view allows to specify the point of view using translation, rotation, and scale primitives. The model includes a line extending the needle and a line from its tip to the center of the target. The needle should be set in the direction where these two lines meet. One problem with this view is the need of mouse interaction, which is usually not practical.

- Needle view: The user can see the needle and the target, in the direction of the needle. This is especially intuitive for directing the needle.

- Augmented camera view: This view includes a camera video. The camera, like the US probe and the needle, is tracked and calibrated, so it is possible to superimpose the virtual models on top of the video. This way, the point 
Fig. 1. Coordinate systems and transformations (left), views of the needle placement system: ultrasound, world interactive, needle, augmented camera view (right)

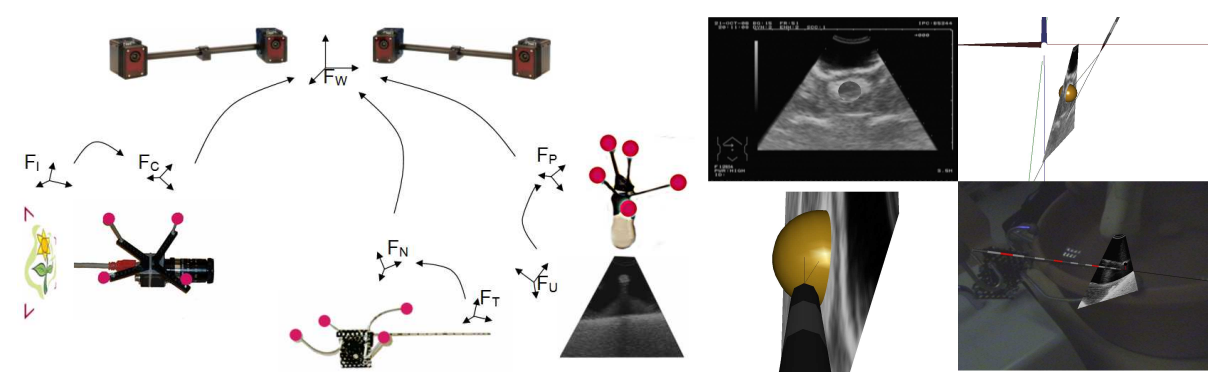

of view is defined by the camera. The main advantage of this view is the ability to integrate other, non tracked elements into the environment. It also helps to qualitatively evaluate the accuracy of the system.

\section{Evaluation of the system}

For testing the overall US navigation accuracy, we submerged the RF needle in water and scanned its tip with the US transducer. When the US image containing the tip was found, the image along with all tracking information was stored and the RF needle's tip was marked manually in the US image. US pixel coordinates were scaled back to millimeters. The needle tip's coordinates in the tracking coordinate frame were transformed into the US plane coordinate system, so both the marked and the tracked coordinates are in the same US plane coordinate system. The Euclidean distance from the marked tip to the tracked tip was calculated and considered as error. We repeated this experiment for 7 times to obtain a more significant error distribution.

\section{Results}

During US calibration we obtained a root mean square error of $1.96 \mathrm{~mm}$ for the needle tip reconstruction accuracy. However, it was difficult to find the needle tip in the US images due to US noise.

To apply the calibration results to a patient, speed of sound correction needs to be incorporated into the calibration procedure, since US machines are usually calibrated to the speed of ultrasound in tissue $(1540 \mathrm{~m} / \mathrm{s})$ and not to its speed in water at room temperature of $21^{\circ} \mathrm{C}$ (ca. $\left.1485 \mathrm{~m} / \mathrm{s}\right)$ [11].

\section{Conclusion}

The surgical navigation system presented in this paper is stable and easy to use. It helps to simplify the RF needle placement by dividing it into two simple consecutive tasks: lesion finding and needle placement. The surgeon does not have 
to simultaneously navigate both the US probe and the RF needle. Additionally, the needle can always be visualized relatively to frozen or live US images.

Since the overall system accuracy mainly relies on an accurate US calibration, we will investigate in further development of an accurate evaluation phantom to validate the US reconstruction accuracy. The navigation system can be improved by adding features such as automatic registration with preoperative data and more complex target modeling, including deformable tissue and anatomic features. This way, the system would help not only to find the target point in the body but also to select a safe path.

\section{References}

1. Gazelle GS, Goldberg SN, Solbiati L, Livraghi T. Tumor ablation with radio frequency energy. Radiology 2000;217:633-646.

2. Prager RW, Rohling RN, Gee AH, Berman L. Rapid calibration for 3-D freehand ultrasound. Ultrasound in Medicine and Biology 1998;24(6):855-869.

3. Mercier L, Lang $\varnothing \mathrm{T}$, Lindseth F, Collins DL. A review of calibration techniques for freehand 3-D ultrasound systems. Ultrasound in Medicine and Biology $2005 ; 31(4): 449-471$.

4. Lang $\varnothing$ T. Ultrasound Guided Surgery: Image Processing and Navigation. Ph.D. thesis. Norwegian University of Science and Technology; 2000.

5. Howard MH, Nelson RC, Paulson EK, Kliewer MA, Sheafor DH. An electronic device for needle placement during sonographically guided percutaneous intervention. Radiology 2001;218:905-911.

6. Khamene A, Vogt S, Azar F, Sielhorst T, Sauer F. Local 3D reconstruction and augmented reality visualization of freehand ultrasound for needle biopsy procedures. In: Procs MICCAI; 2003. 344-355.

7. Sielhorst T, Feuerstein M, Traub J, Kutter O, Navab N. CAMPAR: A software framework guaranteeing quality for medical augmented reality. Int J Comp Assist Radiol Surg 2006;1(Supplement 1):29-30.

8. Fischler MA, Bolles RC. Random sample consensus: A paradigm for model fitting with applications to image. Analysis and Automated Cartography, Commun ACM $1981 ; 24(6)$.

9. Treece GM, Gee AH, Prager RW, Cash CJC, Berman LH. High-definition freehand 3-D ultrasound. Ultrasound in Medicine and Biology 2003;29(4):529-546.

10. Hsu PW, Prager RW, Gee AH, Treece GM. Rapid, easy and reliable calibration for freehand 3D ultrasound. University of Cambridge, Department of Engineering. Trumpington Street, Cambridge CB2 1PZ; 2005.

11. Marczak W. Water as a standard in the measurements of speed of sound in liquids. Acoustical Society of America Journal 1997;102:2776-2779. 